\title{
The Pointy End of Point-of-Care Testing for Direct Oral Anticoagulants
}

\author{
Emmanuel J. Favaloro ${ }^{1,2}$ Giuseppe Lippi ${ }^{3}$ \\ ${ }^{1}$ Department of Haematology, Institute of Clinical Pathology and \\ Medical Research (ICPMR), NSW Health Pathology, Westmead \\ Hospital, Westmead, NSW, Australia \\ 2 Sydney Centres for Thrombosis and Haemostasis, Westmead \\ Hospital, Westmead, NSW, Australia \\ ${ }^{3}$ Section of Clinical Biochemistry, University of Verona, Verona, Italy \\ Thromb Haemost 2020;120:11-13.
}

Direct oral anticoagulants (DOACs), also sometimes called 'NOACs' (variably for novel, new, or non-vitamin K antagonist oral anticoagulants), represent an increasingly applied form of anticoagulant therapy for an ever-expanding range of indications. ${ }^{1,2}$ Routine haemostasis (coagulation) assays are variably sensitive to DOACs, ${ }^{3,4}$ and cannot be reliably used for measuring their concentration. Although routine monitoring of DOACs is not generally required, there are many plausible situations in which knowledge of patient's anticoagulant status may be clinically useful, including unconscious patients presenting as a trauma and bleeding, or patients presenting for emergency surgery, known or unknown to be on an anticoagulant, as well as patients developing thrombosis while on treatment and women with possible pregnancy whereby the effect of these drugs on the foetus is still unknown. ${ }^{3,4}$ Accordingly, sensitive and specific methods for assessing DOACs are increasingly employed in clinical practice. DOACs mainly segregate into one of two agent classes, namely anti-thrombin (anti-activated factor II; anti-FIla, such as dabigatran) and anti-activated factor X (anti-FXa, such as rivaroxaban, apixaban, edoxaban). Like most tests of haemostasis, laboratory-based assays typically utilize citrate anticoagulated plasma to measure DOAC activity. ${ }^{3,4}$ The most commonly applied tests respectively are laboratory-performed direct thrombin inhibitor and chromogenic anti-FXa assays. ${ }^{3,4}$

Point-of-care (POC) tests represent an additional strategy for measuring DOACs. Proponents of POC testing cite faster test times at 'bedside', potentially useful for emergency or surgical use where despatch to a laboratory and availability of finally issued results may exceed clinical desirability. Sometimes, specific laboratory tests for DOACs may also only be available on restricted days or times or at central sites. There are several current and emerging strategies for DOAC testing, including POC. ${ }^{3-11}$ However, the research

received

September 23, 2019

accepted

September 23, 2019

Address for correspondence Emmanuel J. Favaloro, PhD, FFSc, ICPMR, NSW Health Pathology, Westmead Hospital, Westmead, NSW 2145, Australia (e-mail: emmanuel.favaloro@health.nsw.gov.au).

group headed by Harenberg has taken a unique strategy to develop a POC for DOAC testing using urine as the biological material and a method they have called 'DOAC Dipstick. ${ }^{5}$ The basis for the test is that some 30 to $80 \%$ of DOACs are excreted into the urine, and that sensitive and specific tests for either anti-FIla or anti-FXa in urine can therefore be developed. With this assay, the dipstick contains a matrix where specific enzymes and substrates directed against DOACs are immobilized to enable detection of both types of agents (anti-FIla or anti-FXa) on the same dipstick, at the same time, but using separate pads. The dipstick also contains additional pads to control for colour of urine and assess urine creatinine concentration, which respectively help to avoid false positives and identify impaired renal function.

It is therefore of interest that the Harenberg group have published the results of a multicentre trial on the methodology in the current issue of the journal. ${ }^{11}$ This report provides important confirmatory evidence on the reliability of this POC system to specifically identify (or exclude) the presence of current anti-FIla or anti-FXa DOACs in urine, with different operators and remarkably high negative and positive predictive value. This article represents the last in a series of publications by the authors around this technique, ${ }^{5,11}$ and importantly identifies a milestone in this journey, initially conceived as an idea in 2013. ${ }^{12,13}$

Like all journeys related to the field of haemostasis, this will no doubt be one that continues. 'Stagnation' reflects a dangerous term in haemostasis. Pathophysiologically, stagnation in blood flow may lead to thrombosis according to the classical Virchow's triad. Metaphorically, stagnation in innovation leads to a separate kind of arrest, namely that of progress. The journey of anticoagulation therapy is itself interesting and on-going. DOACs represent the new kid on the antithrombotic block, with four such agents now imbedded in clinical practice, but several others are potentially

(c) 2020 Georg Thieme Verlag KG Stuttgart · New York
DOI https://doi.org/ $10.1055 / \mathrm{s}-0039-1700874$. ISSN 0340-6245. 


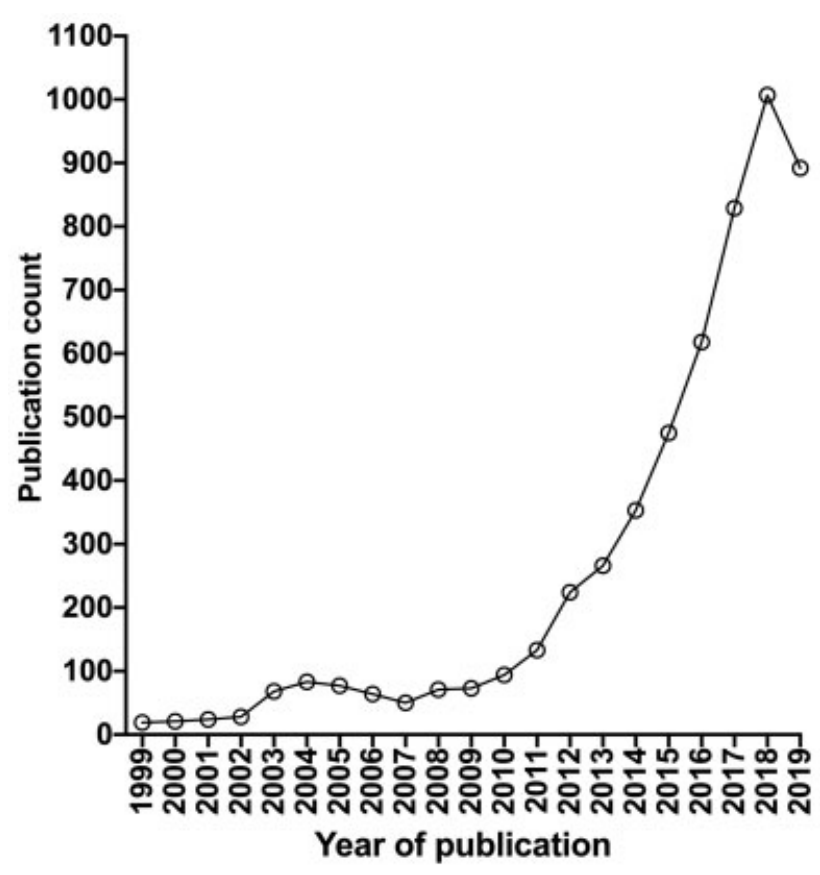

Fig. 1 The ever-expanding literature involving direct oral anticoagulants (DOACs). A simple PubMed search of 'DOAC', 'NOAC' and 'direct oral anticoagulants' (even recognizing that this would not capture all possible derivations of DOACs) identified over 5,600 entries, with the growth over the past 20 years identified here. The count for 2019 represents only a partial year (up to 17th September).

emerging. ${ }^{14}$ A PubMed search of 'DOAC', 'NOAC' and 'direct oral anticoagulants' (albeit recognized to not capture all possible derivations of DOACs) identifies over 5,600 entries, with the explosion in recent entries evident in - Fig. 1. Several such papers have recently appeared in this journal, but perhaps most relevant to the current in focus publication of Harenberg and team, are those related to tests performed for DOACs, or otherwise adversely affected by DOACs.

One standout in particular is the recent International Council for Standardization in Haematology recommendations for laboratory measurement of DOACs, ${ }^{3}$ which also recognize the availability of POC tests such as DOAC Dipstick. As already mentioned, several papers have also been recently published around other novel approaches to DOAC testing. ${ }^{7-10}$

Along with clinical benefits of DOACs come some negative issues around 'test interference'. ${ }^{15-18}$ For example, tests used in investigating lupus anticoagulant (LA), especially the activated partial thromboplastin time and the dilute Russell viper venom time, are very sensitive to the presence of all DOACs, ${ }^{4,15,16}$ and may lead to both false positive and false negative test results for LA, ${ }^{16}$ although this effect can be abrogated by using various DOAC 'neutralisers'. ${ }^{16,17}$ DOACs may also interfere with other tests such as anti-thrombin assays. ${ }^{4,18}$ In this case, the interference is class-specific, as anti-FIla agents interfere with antiFIla-based anti-thrombin assays and anti-FXa agents interfere with anti-FXa-based anti-thrombin assays.

In conclusion, DOACs currently represent the main class of clinical anticoagulants, surpassing the use of vitamin K antagonist therapy in many geographic and clinical settings. ${ }^{1,2}$ Although they do not require routine monitoring by DOAC testing, there may be occasions in which testing may be useful and drive a clinical response that depends on whether or not DOACs are identified, or if high levels of DOACs are identified, and so on. ${ }^{3,4}$ DOAC Dipstick testing using urine represents one strategy for assessing the presence or absence of DOACs quickly and accurately, and using a potentially less invasive technique, also overcoming some potential pre-analytical limitations of drawing blood (i.e. patients with small or difficult veins, haemolysis, sample contamination, sample stability, etc.). ${ }^{5,11-14}$ Some additional aspects of the robustness of the test may also have to be clarified in due course, such as the impact of urine dilution or concentration, the potential interference of urine components (i.e. erythrocytes, leukocytes, microorganisms, casts) as well as from other substances and drugs excreted with the urine. Naturally, there are also other emerging strategies, ${ }^{6-10}$ and the goal-posts may also be shifting with emergence of even 'newer' DOACs. ${ }^{14}$ Finally, strategies to test for DOACs may become eventually surpassed by strategies to permit other haemostasis tests to be performed without inadvertent interference of DOACs, given especially that performance of 'thrombophilia tests' is inevitable on patients on anticoagulant therapy. ${ }^{15-18}$

\section{Note}

The views expressed in this editorial are those of the authors, and are not necessarily those of NSW Health Pathology or the University of Verona.

\section{Conflict of Interest}

None declared.

\section{References}

1 Lippi G, Mattiuzzi C, Adcock D, Favaloro EJ. Oral anticoagulants around the world: an updated state-of the art analysis. Ann Blood 2018;3:49

2 Favaloro EJ, Pasalic L, Lippi G. Replacing warfarin therapy with the newer direct oral anticoagulants, or simply a growth in anticoagulation therapy? Implications for pathology testing. Pathology 2017;49(06):639-643

3 Gosselin RC, Adcock DM, Bates SM, et al. International Council for Standardization in Haematology (ICSH) recommendations for laboratory measurement of direct oral anticoagulants. Thromb Haemost 2018;118(03):437-450

4 Favaloro EJ, Pasalic L, Curnow J, Lippi G. Laboratory monitoring or measurement of direct oral anticoagulants (DOACs): advantages, limitations and future challenges. Curr Drug Metab 2017;18(07): 598-608

5 Harenberg J, Schreiner R, Hetjens S, Weiss C. Detecting anti-IIa and anti-Xa direct oral anticoagulant (DOAC) agents in urine using a DOAC Dipstick. Semin Thromb Hemost 2019;45(03): 275-284

6 Ansell J, Zappe S, Jiang X, et al. A novel whole blood point-ofcare coagulometer to measure the effect of direct oral anticoagulants and heparins. Semin Thromb Hemost 2019;45(03): 259-263

7 Bluecher A, Meyer Dos Santos S, Ferreirós N, et al. Microfluidic coagulation assay for monitoring anticoagulant therapy in acute stroke patients. Thromb Haemost 2017;117(03):519-528

8 Poli S, Härtig F, Spencer C, et al. Diagnostic accuracy of a novel chromogenic direct thrombin inhibitor assay: clinical experiences for dabigatran monitoring. Thromb Haemost 2017;117(12): 2369-2375 
9 Harder S, Santos SMD, Krozer V, Moll J. Surface acoustic wavebased microfluidic coagulation device for monitoring anticoagulant therapy. Semin Thromb Hemost 2019;45(03):253-258

10 Kaikita K, Hosokawa K, Dahlen JR, Tsujita K. Total ThrombusFormation Analysis System (T-TAS): clinical application of quantitative analysis of thrombus formation in cardiovascular disease. Thromb Haemost 2019;19(10):1554-1562

11 Harenberg J, Beyer-Westendorf J, Crowther M, et al. Accuracy of a rapid urine diagnostic test to detect the presence of direct oral factor Xa or thrombin inhibitors - a multicentre trial. Thromb Haemost 2020;120(01):132-140

12 Harenberg J, Krämer S, Du S, Weiss C, Krämer R. Concept of a point of care test to detect new oral anticoagulants in urine samples. Thromb J 2013;11(01):15

13 Harenberg J, Du S, Krämer S, et al. Novel methods for assessing oral direct factor Xa and thrombin inhibitors: use of point-of-care testing and urine samples. Semin Thromb Hemost 2013;39(01):66-71
14 Lippi G, Gosselin R, Favaloro EJ. Current and emerging direct oral anticoagulants: state-of-the-art. Semin Thromb Hemost 2019;45 (05):490-501

15 Antovic A, Norberg EM, Berndtsson M, et al. Effects of direct oral anticoagulants on lupus anticoagulant assays in a real-life setting. Thromb Haemost 2017;117(09):1700-1704

16 Favaloro EJ. The Russell viper venom time (RVVT) test for investigation of lupus anticoagulant (LA). Am J Hematol 2019;94(11): 1290-1296

17 Favaloro EJ, Gilmore G, Arunachalam S, Mohammed S, Baker R. Neutralising rivaroxaban induced interference in laboratory testing for lupus anticoagulant (LA): a comparative study using DOAC Stop and andexanet alfa. Thromb Res 2019;180:10-19

18 Rühl H, Reda S, Müller J, Oldenburg J, Pötzsch B. Activated factor Xbased versus thrombin-based antithrombin testing in thrombophilia workup in the DOAC era. Thromb Haemost 2018;118(02): 381-387 